\title{
Evolution of Primate Multilevel Social Systems: Proboscis Monkey Society as Complex System
}

\author{
Ikki Matsuda, Ikuma Adachi, and Hiroki Koda
}

\begin{abstract}
Great apes like chimpanzees often provide referential models to understand evolutional trajectories of human behaviour, cognition, morphology and social system as humans and chimpanzees shared a common ancestor only 5-7 million years ago (Мya). However, there are other lesser known non-human primates which are phylogenetically far to humans, but sharing similar traits with humans in terms of social system, i.e., multilevel societies. Among primate social systems, the multilevel society, in which smaller levels of social organization aggregate into larger units, is one of the most complex, though its origins and function are still poorly understood. Proboscis monkeys (Nasalis larvatus), one of the rare primate species reported multilevel social system, belong to the odd-nosed colobines, and are a large, sexually dimorphic and primarily arboreal species. We will overview what/how multilevel society in proboscis monkeys and discuss proximate mechanisms maintaining and the selective factors underlying their social system. Investigation on primate multilevel social systems would not only provide insights into the evolutionary history of human social system but also possibility develop our understanding how brain encodes the spatial position of others in such a complex society.
\end{abstract}

\footnotetext{
I. Matsuda $(\bowtie)$

Chubu University Academy of Emerging Sciences, 1200, Matsumoto-cho,

Kasugai-shi, Aichi 487-8501, Japan

e-mail: ikki-matsuda@isc.chubu.ac.jp
}

Wildlife Research Center of Kyoto University, Kyoto, Japan

Japan Monkey Centre, Inuyama, Japan

Institute for Tropical Biology and Conservation, Universiti Malaysia, Sabah, Malaysia

I. Adachi $\cdot$ H. Koda

Primate Research Institute, Kyoto University, Inuyama, Aichi 484-8506, Japan

e-mail: ikuma.adachi@gmail.com

H. Koda

e-mail: koda.hiroki.7a@kyoto-u.ac.jp

A. Lintas et al. (eds.), Advances in Cognitive Neurodynamics (VII), Advances in Cognitive Neurodynamics,

https://doi.org/10.1007/978-981-16-0317-4_35 